\title{
The Empirical Research on the Continuous Usage Intention of Mobile Internet Users
}

\author{
Jinbo Wang \\ School of Economics, Xiamen University, Xiamen 361005, China; \\ Wangjb168@163.com
}

Keywords: Mobile Internet, continuous usage intention, empirical research.

\begin{abstract}
In recent years, the Mobile Internet has become the focus of competition in the global information industry. This paper studies the main factors influencing the consumers continue to use the Mobile Internet, collect relevant data by using the questionnaire method, and analysis of the data by EVIEWS; finally, combined with the statistical results of comments on operators and mobile phone terminal services.
\end{abstract}

\section{Introduction}

Mobile Internet is the new generation of open network which core technology is a broadband IP, and it can provide voice, fax, data, images, multimedia and other high-quality communications services. The core of the Mobile Internet is the Internet, applications and content is still the fundamental of Mobile Internet. Mobile Internet has the characteristics of real-time, privacy, portability, accuracy, and can be positioned, and increasing smart mobile devices is one of the important features of the Mobile Internet.

\section{Design and implementation of questionnaire}

\subsection{Influencing factors of consumer behavior intention in Mobile Internet.}

In this paper, we study the usage intention of Mobile Internet. Only to meet the needs of consumers, it can promote the sustainable and healthy development of the Mobile Internet, and it also is the source of the interests of all enterprises.

The influence factors on consumer behavior intention of Mobile Internet include: network quality, perceived entertainment, perceived price, perceived risk, perceived usefulness, perceived ease of use, social influence, and these variables will be set as independent variables. While the consumer's continuous usage intention of Mobile Internet as the dependent variable.

2.2 The measurement index of variables and the theoretical hypothesis.

The variables involved in this research are network quality, perceived entertainment, perceived price, perceived risk, perceived usefulness, perceived easy-using, social impact, and continuous usage intention. Following on these variables given the measurement items, each item are used Likert5 scale, from strongly disagree to strongly agree: strongly disagree $=1$, disagree $=2$, neither agree nor disagree $=3$, agree $=4$, strongly agree $=5$.

Network quality can be measured from the connection quality, connection speed, and information quality. Network quality is the key factor to determine whether users use Mobile Internet.

Perceived entertainment refers to the interesting and intrinsic enjoyment of the user by using Mobile Internet. It includes personal feelings, personal attention and interaction of Mobile Internet.

Perceived price refers to the perception of the cost which users to experience an application in the Mobile Internet.

Perceived risk refers to the negative results which to be inconsistent with expectations may occur in the process of using the Mobile Internet, and if the negative results occur, the user's psychological expectations for the severity of the results. This study mainly measures the user's perception of privacy risk, and the loss property. 
Perceived usefulness refers to the user experience of the utility of the Mobile Internet services, including the effectiveness of its games, music and other functions. Perceived usefulness is an important variable in the continuous using research model.

Perceived ease-using refers to consumers perceive the ease of use of Mobile Internet, including perceived ease of use and complexity. When the Mobile Internet is easier to set up and use, consumers will be more confident on self-efficacy and self-control, and their attitude towards Mobile Internet will be more active.

Social impact refers to Mobile Internet users aware of the social principle of thoughts, feelings and actions, which to be able to impact on their willing to continuous use the Mobile Internet. It includes the subjective norm and the image. Subjective norm refers to the users satisfy expectations of the group in order to gain appreciation or avoid punishment. Image refers to perceived use of innovation to improve the personal image or status in the social system.

Continuous usage intention refers to the user, will further take certain actions after a period of time of the Mobile Internet service experience, such as the possibility of continuous usage, positive recommendations or renew more Mobile Internet services.

Table 1 Influencing factors on behavior intention of Mobile Internet users

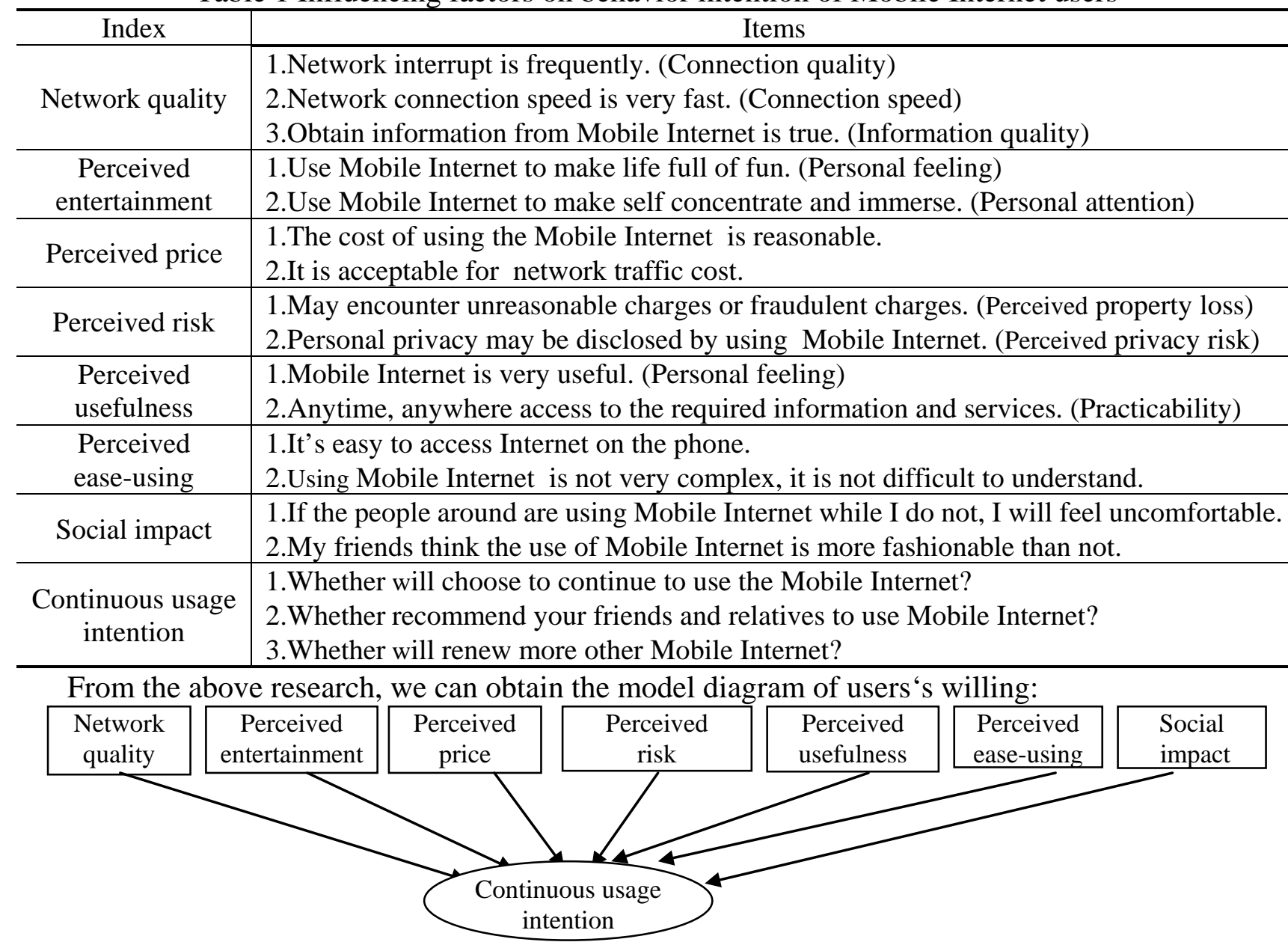

Fig. 1 The model of the users's continuous usage intention

According to the above model, this study put forward the following hypothesises:

Hypothesis 1:High network quality will positively affect the users's continuous usage intention;

Hypothesis 2:Perceived entertainment will positively affect the users's continuous usage intention;

Hypothesis 3: Perceived price will negatively affect the users‘s continuous usage intention;

Hypothesis 4: Perceived risk will negatively affect the users's continuous usage intention;

Hypothesis 5: Perceived usefulness will positively affect the users's continuous usage intention;

Hypothesis 6: Perceived ease-using will positively affect the users's continuous usage intention; 
Hypothesis 7: Social impact will positively affect the users‘s continuous usage intention; 。

\section{Empirical analysis}

\subsection{Descriptive statistics analysis.}

We collected our data by the means of questionnaires from the $10^{\text {th }}$ Nov to the $15^{\text {th }}$ Nov, 2015 . We sent out 340 questionnaires, and 213 valid questionnaires were collected with validity rate $62.6 \%$.

Firstly, a descriptive statistical analysis was conducted on the valid questionnaires, and the result was shown in the following table.

Table 2 Characteristic statistics of Mobile Internet users

\begin{tabular}{c|c|c|c}
\hline Statistical variables & Category & Number & Percentage \\
\hline \multirow{3}{*}{ Average communication cost } & $<$ 30yuan & 16 & $7.5 \%$ \\
monthly & 30-50yuan & 72 & $33.8 \%$ \\
& $50-100$ yuan & 94 & $44.1 \%$ \\
& $>=100$ yuan & 31 & $14.6 \%$ \\
\hline \multirow{3}{*}{ Average time on Mobile } & $<1 \mathrm{Hr}$ & 68 & $31.9 \%$ \\
Internet daily & $1-2 \mathrm{Hr}$ & 60 & $28.2 \%$ \\
& $2-4 \mathrm{Hr}$ & 42 & $19.7 \%$ \\
\hline \multirow{2}{*}{ Content of access Mobile } & $>=4 \mathrm{Hr}$ & 43 & $20.2 \%$ \\
\hline Internet & games, music, video and other entertainment & 46 & $21.6 \%$ \\
& Micr-blog, WeChat, QQ, and other media & 156 & $73.2 \%$ \\
& email, shopping, travel and other practicability & 11 & $5.2 \% \%$ \\
\hline
\end{tabular}

From the average communication cost monthly, more concentrated in the 30-50 yuan and 50-100 yuan. From average time on the internet daily, one hour or less accounted for 31.9\%, the proportion of others almost same. From the content on Mobile Internet, for media content accounted for $73.2 \%$, followed by entertainment $21.6 \%$.

Table 3 Information of Mobile Internet users (five points)

\begin{tabular}{c|c|c|c|c|c|c|c|c|c}
\hline Index & \multicolumn{3}{|c|}{ Network quality } & \multicolumn{2}{c|}{ Perceived entertainment } & \multicolumn{2}{c}{ Perceived usefulness } & \multicolumn{2}{c}{ Perceived risk } \\
\hline Items & 1 & 2 & 3 & 1 & 2 & 1 & 2 & 1 & 2 \\
\hline Point & 2.82 & 2.73 & 2.95 & 3.64 & 3.25 & 3.93 & 3.97 & 3.91 & 3.92 \\
\hline Index & \multicolumn{2}{|c|}{ Perceived price } & \multicolumn{2}{c|}{ Perceived ease-using } & \multicolumn{2}{c}{ Social impact } & \multicolumn{2}{c}{ Continuous usage intention } \\
\hline Items & 1 & 2 & 1 & 2 & 1 & 2 & 1 & 2 & 3 \\
\hline Point & 2.70 & 2.82 & 3.79 & 3.53 & 2.76 & 2.84 & 3.70 & 3.28 & 2.74 \\
\hline
\end{tabular}

From table 3, network quality have not reached 3 points which shows that the user is not very satisfied with it; from perceived entertainment, the users of Mobile Internet reached the average level, looks like the users still feel good, and that Mobile Internet is very good for their life; from perceived risk, the users sometimes still worry about that their interests have been violated in the use of Mobile Internet; from perceived price, the price level is not reached the range of consumers can accept; from perceived ease-using, user interface of mobile phone is very good to get started for users; from continuous usage intention, the prospects for Mobile Internet still has very large development space.

\subsection{Model parameter analysis.}

We analyze the impact of various factors on continuous usage intention by EVIEWS, and get the relationship between the various factors and continuous usage intention. Regression coefficients, related statistics, and t-value values are shown in table 4 . The goodness of fit of the regression is 0.437 , and the goodness of fit is 0.415 after revise. 
Table 4 Model parameter analysis

\begin{tabular}{c|c|c|c|c}
\hline Hypothesis & Regression coefficients & t-value & significant & Result \\
\hline Hypothesis 1 & 0.329 & 3.777 & Yes & Accept \\
\hline Hypothesis 2 & 0.135 & 1.850 & Yes & Accept \\
\hline Hypothesis 3 & 0.157 & 2.342 & Yes & Accept \\
\hline Hypothesis 4 & -0.067 & -1.670 & Yes & Accept \\
\hline Hypothesis 5 & 0.134 & 2.547 & Yes & Accept \\
\hline Hypothesis 6 & 0.079 & 1.087 & No & Reject \\
\hline Hypothesis 7 & 0.084 & 1.723 & Yes & Accept \\
\hline
\end{tabular}

Removing the effect of perceived ease-using on continuous usage intention, we can sort the descending order by the regression coefficient: network quality, perceived usefulness, perceived entertainment, perceived price, social impact, perceived risk.

\section{Summary}

This study verified the correlation between network quality, perceived entertainment, perceived usefulness, perceived risk, perceived price, social impact with continuous usage intention of Mobile Internet users by empirical research, and to understand the strength of effects of each factor on continuous usage intention. At the same time we sort the variables according to the strength of effects: network quality, perceived usefulness, perceived entertainment, perceived price, social impact, perceived risk, and these variables are the priority among priorities in the future development process of Mobile Internet.

At last, it can provide the following recommendations to the operator and the mobile terminal according this study result:

(1) In order to enrich the function of the Mobile Internet, it is necessary to design the specific needs of different groups of users; at the same time should try to expand the Mobile Internet business coverage, to connect the business and the user's work and life together, to provide the information needed for consumers at the maximum extent, so that it can let consumers truly feel the convenience and benefits of Mobile Internet, and may have the biggest entertainment.

(2) Operators must be clearly identified in the extra charge or abnormal consuming traffic, not do unreasonable charges to the consumer. At same time, mobile phone payment must have security, to establish and maintain the safety and integrity of the Mobile Internet environment which can increase the trust of consumers. So consumers will dare to subscribe to more business on the basis of trust and each side both can reached the own purpose.

(3) To improve network quality, web link speed, and search ability, and to reduce the page error rate, so that can reduce the time consumption.

\section{Acknowledgement}

This research is supported by Philosophy and Social Science Planning Project of Fujian Province, China (Grant FJ2016B175), Fundamental Research Funds for the Central Universities of China (Grant 20720151029), and General project of education and scientific research of young and middle-aged teachers in Fujian Province (Grant JAS14013).

\section{References}

[1]. Lu Min. On the current situation and development trend of Mobile Internet in China Ziqing Hou. China Science and Technology Information. 2015.1.

[2]. Yoo-Kyoung, S., Lauren R.B. The influence of college students'shoppng orientation and gender differences on online information searches and purchase behaviours. International Journal of Consumer Studies.2008,32(2):113-128 
[3]. Xiang Li. Study on consumers Mobile Internet Information search behaviour and influencing Factors (Master, South China University of Technology, 2011).

[4]. Weihua He. A review of the research theory of customer repeat purchase intention. Contemporary Economic. 2007(20):142-144.

[5]. Dawei Zhang. Eviews data statistics and analysis tutorial. Tsinghua university press, 2010. 